\title{
Subtotal Vaginectomy as a Treatment for Vaginal Tumours in Two Bitches
}

\author{
Cornel IGNA ${ }^{1 *}$, Daniel BUMB ${ }^{1}$, Adelina PROTEASA ${ }^{1}$, Roxana DASCALU ${ }^{1}$, Larisa SCHUSZLER ${ }^{1}$, \\ Violeta IGNA ${ }^{1}$, and Diana BREZOVAN ${ }^{1}$ \\ ${ }^{1}$ Banat's University of Agricultural Science and Veterinary Medicine, "King Mihai I of Romania" from \\ Timisoara, Romania \\ ${ }^{*}$ Corresponding author, e-mail: ignacornel@gmail.com
}

Bulletin UASVM Veterinary Medicine 73(1)/2016

Print ISSN 1843-5270; Electronic ISSN 1843-5378

doi:10.15835/buasvmcn-vm: 11933

\begin{abstract}
Leiomyoma/leiomyosarcoma, fibroma, and transmissible venereal tumor account for most of the canine vulvar/vaginal neoplasms (Thacher and Bradley, 1983). Genital tract tumours are usually seen in medium aged non-spayed dogs and the recommended treatment choice is the surgery (Klein, 2001). Vaginectomy can be complete or subtotal. The indication for a vaginectomy is for the treatment of large or malignant vaginal tumours (Tivers and Baines, 2012).

To report a surgical technique for subtotal vaginectomy using a combined abdominal and vestibular approach; postoperative management; and short-term outcome.

Two female dogs were diagnosed with extensive intramural caudal vagina and cervix mass and pyometra. Preoperative evaluation and a centesis biopsy were performed. Through a ventral median celiotomy the ovariohysterectomy was performed. A subtotal vaginectomy was carried out using an episiotomy approach. The caudal part of the vagina was dissected and separated from the vestibule at the vestibule-vaginal junction, just cranial to the urethral orifice and the vagina freed from any remaining pelvic attachments. Dogs were reexamined at one month and 6 months postoperatively.

A combined abdominal and vestibular approach permitted subtotal vaginectomy in both cases. Histopathological examination of the excised tumour reveals fibroleiomyoma, squamous carcinoma and haemangioma. The two bitches included in this study showed a satisfactory outcome with no tumour recurrence or metastases at follow-up examination.

The surgical technique described here allowed complete resection of extensive vaginal lesions, was not associated with major complications, and outcome was favorable.
\end{abstract}

Keywords: bitch, fibroleiomyoma, haemangioma, squamous carcinoma, vaginectomy

\section{INTRODUCTION}

Vaginal neoplasia is relatively common in the bitch. The most common tumour is leiomyoma, lipomas, fibromas, and transmissible venereal tumor (Thacher and Bradley, 1983). Genital tract tumours are usually seen in medium aged nonspayed dogs and the recommended treatment choice is the surgery (Klein, 2001).

Vaginal tumours are usually benign (Tivers and Baines 2012). Many of these tumours are pedunculated, often on narrow stalks. In rare cases, vaginal tumours are sessile, broad-based or irregular. They grow in a concentric way, either towards the vestibular area or towards the cervix (Thacher and Bradley 1983; Salomon et al., 2004). Most benign tumors are managed by local resection via episiotomy combined with ovariohysterectomy to prevent recurrence.

Leiomyosarcomas, carcinomas, and transmissible veneral tumors are the most commonly reported malignant neoplasms of the vagina and unlike benign tumors, they tend to be broad based and infiltrative (Thacher and Bradley, 1983; Hill et al., 2000; Tivers and Baines 2012). 
Treatment of malignant vaginal tumors requires total vaginectomy, techniques that combine an episiotomy approach with laparotomy augmented by pubic osteotomy or ostectomy. The prognosis for malignant neoplasia is considered poor due to high rates of recurrence and metastasis (Hill et al., 2000; Tivers and Baines 2012). In bitches, larger retrospective studies dealing with the total vaginectomy technique would be valuable to assess this invasive technique and its potential complications (Salomon et al., 2004).

This report describes two cases of malignant vaginal tumours in bitches; a surgical technique for subtotal vaginectomy using a combined abdominal and vestibular approach; postoperative management; and short-term outcome.

\section{MATERIALS AND METHODS}

Large, broad-based vaginal masses were diagnosed in two intact bitches. Preoperative evaluation consisted of physical examination, vaginal and rectal palpation, a serum chemistry profile and ultrasonographic examination of the entire abdominal cavity,including the lower urogenital tract, and thoracic - abdominal radiographs for evidence of metastasis.

The protocol for general anaesthesia included premedication with diazepam $(0.4 \mathrm{mg} / \mathrm{kg}$ b.w., i.v.) and ketamine ( $5 \mathrm{mg} / \mathrm{kg}$ b.w., i.v.) followed by induction with propofol ( $3 \mathrm{mg} / \mathrm{kg}$ b.w., i.v.). After endotracheal intubation, general anaesthesia was maintained with izoflurane vaporized in oxygen using intermittent positive pressure ventilation. Postoperative analgesia was provided with one

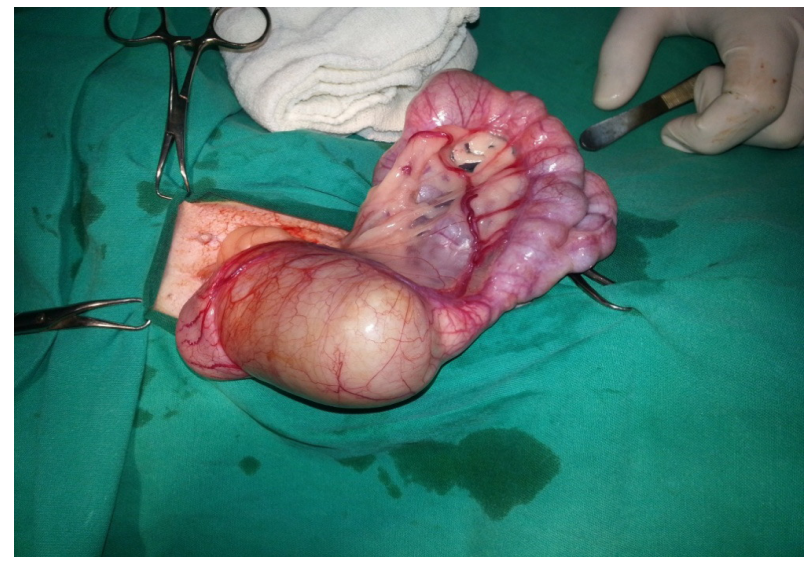

Fig. 1 - Abdominal exposition of the reproductive tract dose of butorphanol ( $0.4 \mathrm{mg} / \mathrm{kg}$ s.c.) administered with 15 minutes before recovery. Ampicillin (20 $\mathrm{mg} / \mathrm{kg}$ i.v.) was administered at induction and at the end of surgery, which lasted two hours.

Abdominal procedure: The dog was positioned in dorsal recumbency. A catheter was aseptically placed into the urethra to facilitate urethral tubercle identification. The ventral midline region, perineal and vulva area were aseptically prepared and a skin incision was made from the umbilicus to the cranial pubic edge. Each bitch has undergone an initial ovariohysterectomy. The procedure was modified, after Salomon et al. (2004), to facilitate a cranial vaginectomy (Fig. 1). The fascial and peritoneal attachments between the vagina and the rectum within the rectogenital pouch were resected. Similarly, were dissected the attachment between the vagina and the urethra within the vesicogenital pouch for avoiding any disruption of the craniolateral aspect of the urethra and the periurethral tissues. The cranial and caudal branches of the vaginal artery and vein were ligated and bluntly dissected of the perivaginal tissues as far caudally as possible (caudal to cervix). A transfixing stay suture with a large loop anchored through all layers of the cranial opening of the vagina was placed and the loop of the suture was passed into the vaginal lumen (Fig. 2 - arrow).

The routine closure of the celiotomy was performed.

Vaginal procedure: After neutering, each bitch was placed in sternal recumbency with the perineum slightly raised. A subtotal vaginectomy was carried out using a vestibular approach

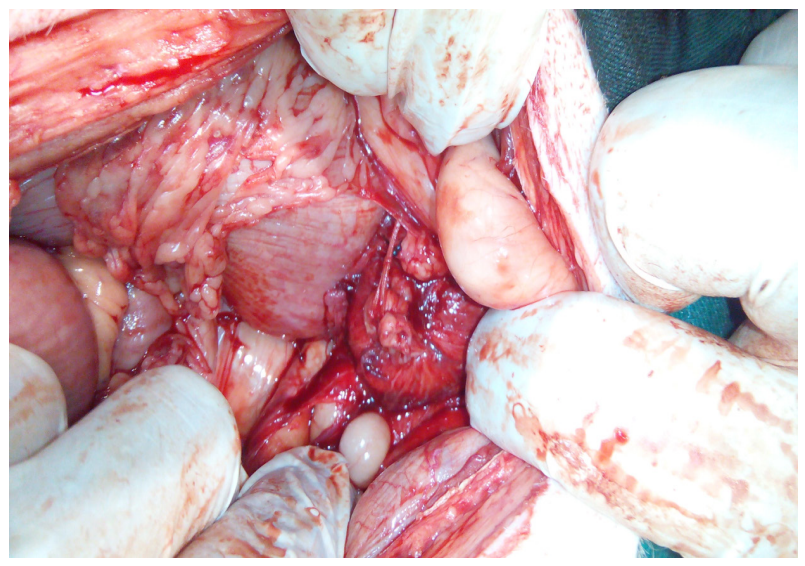

Fig. 2 - A transfixing stay suture of the cranial opening of the vagina 


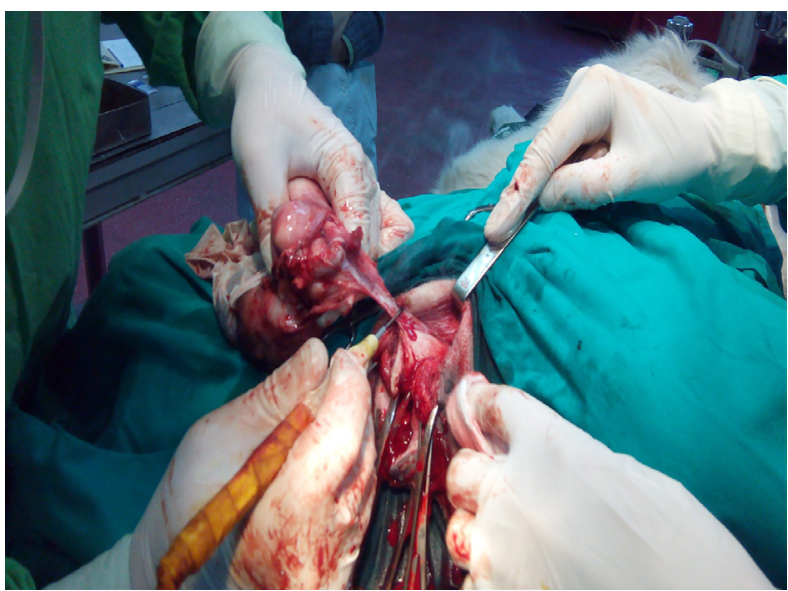

Fig. 3 - Externalised vaginal tumour

through an episiotomy. The loop of the transfixing stay suture was identified in the vaginal lumen and retracted caudally, withdrawing the cranial vagina to the episiotomy site. The caudal part of the vagina was dissected and separated from the vestibule at the vestibulo-vaginal junction, just cranial to the urethral tubercle. The constrictor vestibule muscles were dissected from the vestibule. The dorsal vaginal branches of the vaginal vessels were ligated or electrocauterised. The catheterised urethra was identified, remains untouched. After elevation of the surrounding adhesions, the completely dissected vagina was externalised (Fig 3). The deep pelvic space was obliterate with simple interrupted sutures - PDS II (Fig. 4). The urethra was identified during this procedure to ensure that it is not included.

The episiotomy site was closed routinely in three layers. Vaginal mucosa was apposed with 3 metric polygliycolic acid - PGA (Sentesorb) using interrupted absorbable sutures. The muscular layer was sutured in a continuous pattern using 2 metric polygliycolic acid - PGA (Sentesorb). A simple continuous pattern with 3 metric nylon - Monolon (Sentesorb) was used to close the cutaneous wound.

The urinary catheter was maintained for $48 \mathrm{hrs}$ postoperatively.

Sections of each tumour were submitted for histopathological examination. The fresh cytological specimens were made by printing the tissue samples on slides and stained with DiffQuick. For permanent histological specimens, the collected tissue samples were fixed with

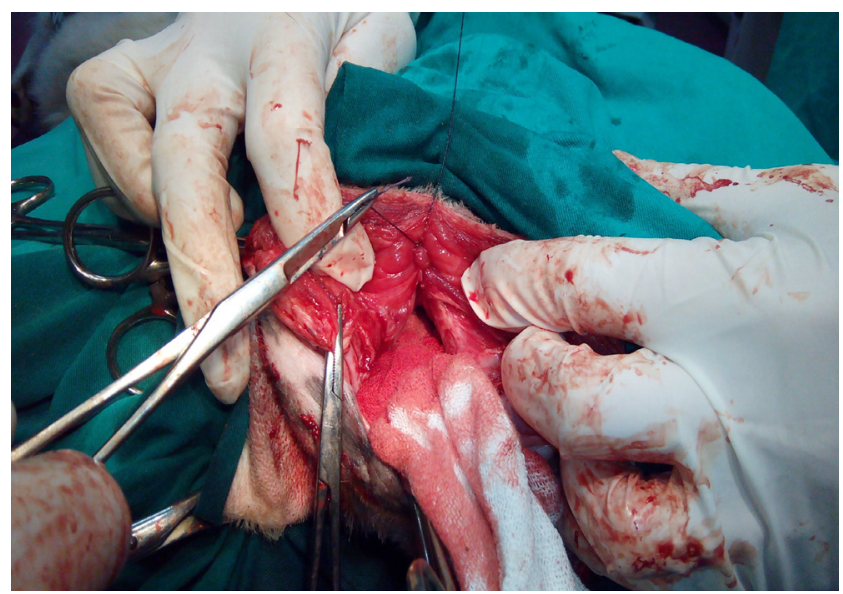

Fig. 4 - The deep pelvic tissues apposed in a simple interrupted sutures

$80 \%$ volume ethyl alcohol. After the embedding procedure with paraffin, the tissue fragments were cut in thin slices, at 5 micrometers and stained by Hematoxilin \& Eosin and Diff-Quick methods.

Dogs were re-examined at one month and 6 months postoperatively.

\section{RESULTS AND DISCUSSION}

A 6-year-old, intact female Bichon Bolognese, weighing $3.5 \mathrm{~kg}$ (case 1) and a 7-year-old, intact female Poodle, weighing $6.5 \mathrm{~kg}$ (case 2) were presented with two-month history of dysuria and vaginal discharge, respectively with constipation and perineal swelling.

The physical examinations showed a haemorrhagic vulval discharge and a perineal enlargement due to a nonmobile vestibular mass, respectively a large mass that involved most of the vagina and vestibule and that deforming the abdominal contour. The exams of the external urethral meatus not showed it to have been invaded by the vaginal mass tumors.

Ultrasonographic examination of the reproductive tract revealed, in the both cases, multinodular masses located in the body and the cervix of the uterus, and extending caudally to the vagina. At Poodle bitch, ultrasonographic and radiographic examinations revealed a heterogeneous mass that compressing the rectum.

The serum chemistry profiles were within normal limits in the both bitches. The chest and abdominal radiographs showed no evidence of metastasis. 
After completion of the ovariohysterectomy, in the both cases a subtotal vaginectomy was carried out using a vestibular approach through an episiotomy. Postoperative examination of the resected tumour confirmed that it had been totally excised (Fig. 5 and 6). There were no recorded postoperative complications; the both bitches were discharged at the 7 day after surgery. The two bitches included in this study showed a satisfactory outcome with no tumour recurrence or metastases at follow-up examination.

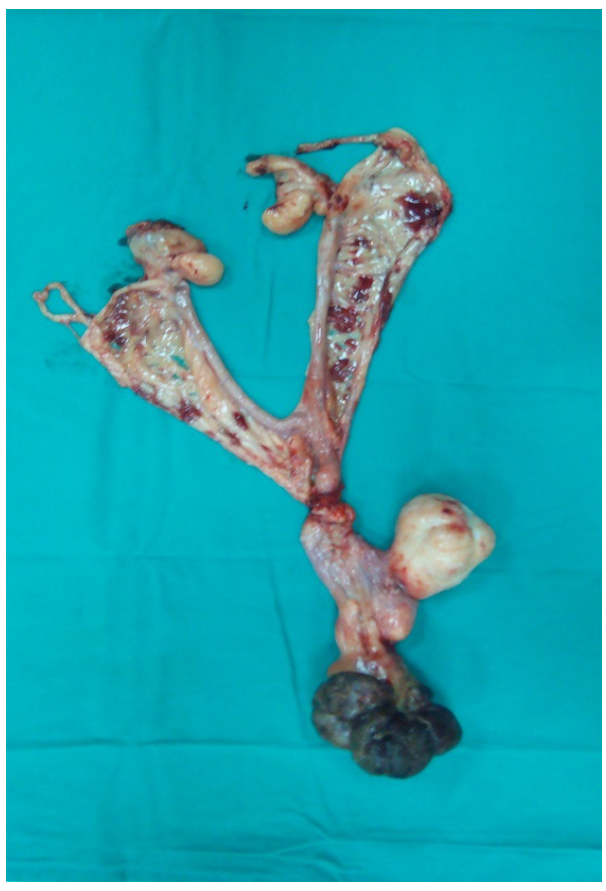

Fig. 5 - A complete reproductive tract resection-case 1

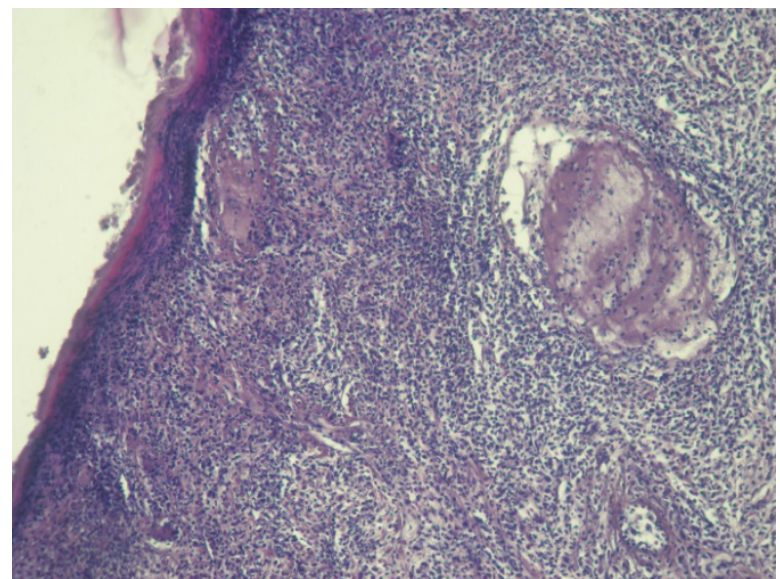

Fig. 7. - Histological section of vaginal tumor: neoplastic cells infiltration, H\&E stain, 100X
In the case 1 histological exams of the massive abnormal tissular fragments collected from the lower part of the genital tract pleads for invasive cervical and vaginal squamous carcinoma in association with hemangioma. Thus, the histological architecture revealed the stromal infiltration with small, spherical neoplastic cells (Fig. 7). Beneath this cellular infiltration, hemangioma was observed (Fig. 8).

Haemangiosarcoma of the vulva and vestibulovagina in the dog is rare, with only two cases were

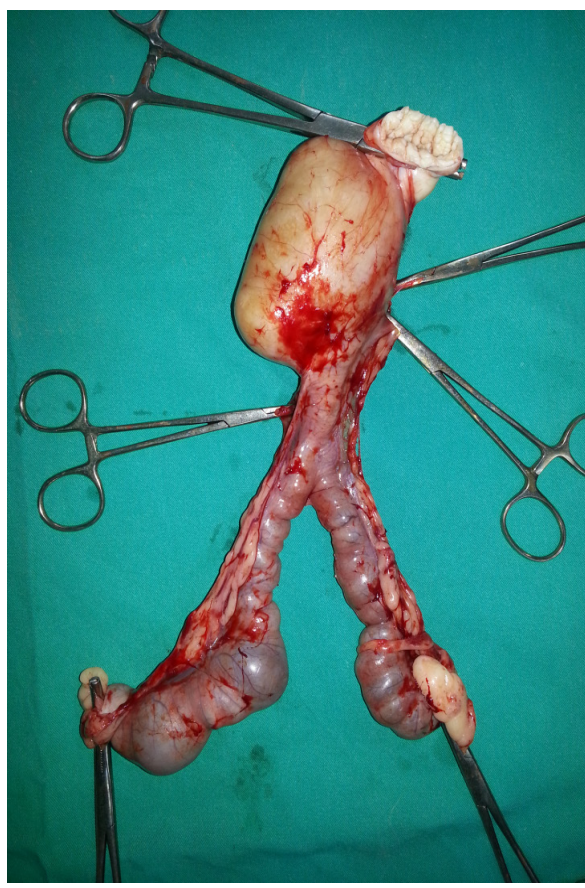

Fig. 6 - A complete reproductive tract resection-case 2

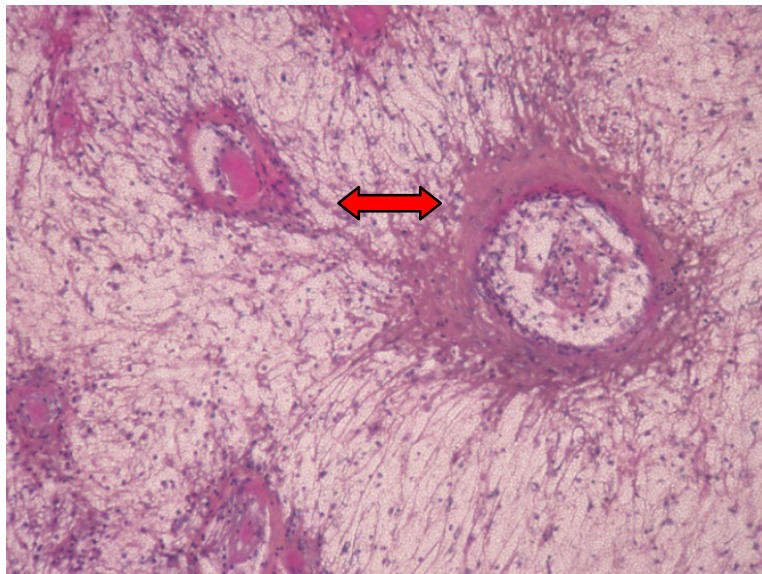

Fig. 8. - Histological section of vaginal tumor: hemangiomas $(\rightarrow)$, H\&E stain, 200X 


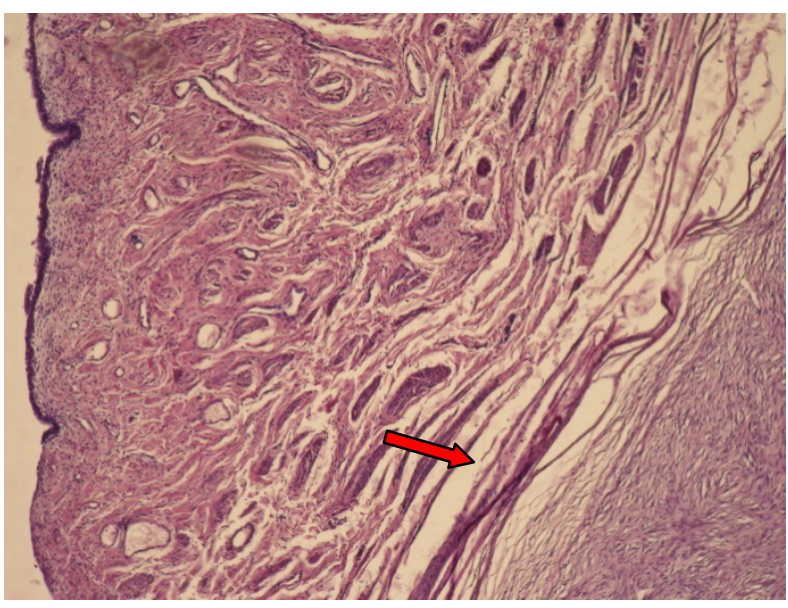

Fig. 9. - Histological section of intramural fibroids: pseudocapsule $(\rightarrow)$, H\&E stain, 100X

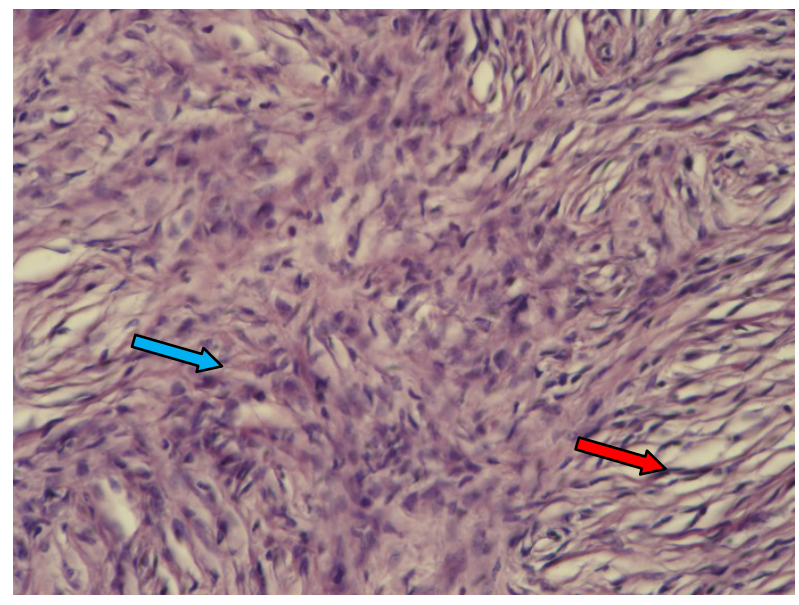

Fig. 10. - Histological section of intramural fibroids: two types of spindle cells: smooth muscle cells-like type $(\rightarrow)$ and fibroblastic cell-like type $(\rightarrow)$, H\&E stain, $400 \mathrm{X}$

1983; Withrow and Susaneck, 1986). In this report are presented a tumoral combination in same case (case 1) - squamous cell carcinoma and hemangiosarcoma, confirmed by histopathological findings.

A great deal of information can be gained by digital vaginal and rectal examination (Tivers and Baines 2012). Thoracic radiography and abdominal ultrasonography are indicated in animals with suspected vaginal neoplasia to check for metastases (Salomon et al., 2004; Tivers and Baines 2012).

Wide resection of fibroleiomyoma (applied to the case 2) with concurrent ovariohysterectomy is the appropriate treatment, with a guarded but favourable prognosis (Thacher and Bradley 1983).

The vaginectomy comprises wide resection of the whole vagina from the cervix to the vestibulovaginal junction (Wykes and Soderberg 1983). This procedure may be performed either by a vestibular approach or by a ventral pelvic approach (accomplished by celiotomy, pubic osteotomy or ostectomy).

Our approach (combined abdominal and vestibular) has enabled a subtotal vaginectomy for management of extensive intramural lesions and was associated with a low incidence of intraoperative and/or postoperative complications. Subtotal vaginectomy is most readily performed using a combined abdominal and vestibular approach without pubic disruption and without urethral resection and urthroplasty. 


\section{CONCLUSION}

A tumoral combination in same case squamous cell carcinoma and hemangiosarcoma - was described.

Combined abdominal and vestibular approach for subtotal vaginectomy allowed complete resection of extensive vaginal lesions, was not associated with major complications, and outcome was favorable.

Acknowledgements. This research work was carried out with the support of the project Dezvoltarea infrastructurii de cercetare, educatie și servicii în domeniile medicinei veterinare si tehnologiilor inovative pentru RO 05, cod SMISCSNR 2669.

\section{REFERENCES}

1. Hill TP, Lobetti RG, Schulman ML (2000). Vulvovaginectomy and neo-urethrostomy for treatment of haemangiosarcoma of the vulva and vagina. J S Afr Vet Assoc 71(4):256-259.

2. Klein MK (2001). Tumours of the female reproductive system, p. 445-454. In: Withrow SJ, MacEwen EG (Eds.). Small Animal Oncology. WB Saunders, Philadelphia.

3. Salomon JF, Deneuche A, Viguier E (2004). Vaginectomy and urethroplasty as a treatment for non-pedunculated vaginal tumours in four bitches. Journal of Small Animal Practice, 45:157-161.

4. Thacher C, Bradley RL (1983). Vulvar and vaginal tumors in the dog: a retrospective study. J Am Vet Med Assoc 183(6):690-692.

5. Tivers M, Baines S (2010). Surgical diseases of the female genital tract. 2 - Vagina and external genitalia. In Practice 32:362-369

6. Withrow SJ, Susaneck SJ (1986). Tumours of the canine female reproductive tract, p. 525-527. In: Morrow D A (ed.) Current therapy in theriogenology (2nd edn).WB Saunders, Philadelphia.

7. Wykes PM, Soderberg SF (1983) Congenital abnormalities of the canine vagina and vulva. Journal of the American Animal Hospital Association 19:995-1000. 